File 2781, 43(4) 2nd Print (9)

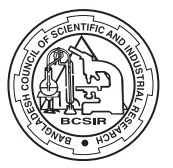

Avaiable online at www.banglajol.info

Bangladesh J. Sci. Ind. Res. 43(4), 521-528, 2008

BCSIR

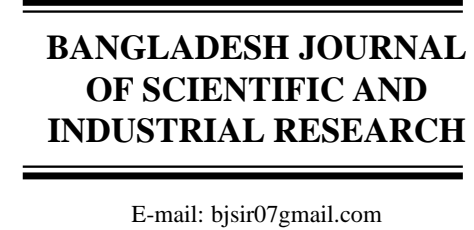

\title{
On the Study of Jacobi Fields in Riemannian Manifolds
}

\author{
Khondokar M. Ahmed \\ Department of Mathematics, University of Dhaka, Dhaka-1000, Bangladesh
}

\begin{abstract}
A new approach of finding a Jacobi field equation with the relation between curvature and geodesics of a Riemanian manifold $M$ has been derived. Using this derivation we have made an attempt to find a standard form of this equation involving sectional curvature $K$ and other related objects.
\end{abstract}

Key words : Riemanign curvature, Sectional curvature, Jacobi equation, Jacobifield.

\section{Introduction}

The concept of differentiating a vector field is not an "intrinsic" geometric notion on $M$. To remedy this state of affairs we consider, instead of usual derivative $\frac{d V}{d t}$, the orthogonal projection $\frac{d V}{d t}$ of on $T_{c(t)} M$. This orthogonal projected vector we call the covariant derivative and it is denoted by $\frac{d V}{d t}$. Jacobi fields are vector fields which is defined by the way of a differential equation which is developed in the study of the exponential mapping (Gauss 1965, Klingenberg 1959 ). The curvature $\kappa(p, \sigma), \sigma \subset T_{p} M$ determines the fastness of the Geodesics. Some aspects of exponential mapping, symmetry property for symmetric connection on a parametrized surface, parametrized surface related with exponential mapping, curvature $R$ of a

*Author for correspondence
Riemannian manifold $M$, sectional curvature, constant sectional curvature, relation between trilinear mapping $R$ and the curvature $R$ will be treated in this present paper (Riemann 1959, Rauch 1953, Myers 1941). Finally we shall find a standard Jacobi equation with a solution.

\section{Preliminaries}

Definition 2.1 An inner product of a manifold $M$ at a point $p \in M$ is a symmetric, bilinear and positive definite form and is denoted by $<$, $>_{\text {p }}$.

If $\psi: U \subseteq \mathbb{R}$ " $\rightarrow M$ is a system of coordinates around $p$ with $\left(\mathrm{x}_{1}, \mathrm{x}_{2} \ldots \ldots, \mathrm{x}_{\mathrm{n}}\right)=q \in \psi(U) \subseteq M$ and $\frac{\partial}{\partial x_{i}}(q)=d \psi_{q}(0, \mathrm{~K}, 1 ; \mathrm{K}, 0$.) then 
$<\frac{\partial}{\partial x_{i}}(q), \frac{\partial}{\partial x_{j}}(q)>_{q}=g_{i j}\left(x_{1}, \ldots, x_{n}\right)$ is a

differentiable function on $U$. We can delete the index $p$ in the function $<_{,}>_{p}$ if there is no confusion.

Definition 2.2 A parametrized curve $\gamma: \mathrm{I} \rightarrow$ $M$ is a geodesic at $t_{0} \in I$ if $\frac{D}{d t}\left(\frac{d \gamma}{d t}\right)=0$ at the point $t_{0}$, if $\gamma$ is a geodesic at $t$, for all, $t \in I$, we say that $\gamma$ is a geodesic. If $[a, b]$ $\subset I$ and $\gamma: \mathrm{I} \rightarrow M$ is a geodesic then the restriction of $\gamma$ to $[a, b]$ is called a geodesic segment joining $\gamma(a)$ to $\gamma(b)$.

The tangent bundle $T M$ is the set of pairs ( $q$, $v), q \in M, v \in T_{q} M$. If $(U, x)$ is a system of coordinates on $M$, then any vector in $T_{q} M, q$ $\in x(U)$, can be written as $\sum_{i=1}^{n} y_{i} \frac{\partial}{\partial x_{i}}$.

Taking $\left(x_{1}, \ldots . x_{n}, y_{1}, \ldots y_{n}\right)$ as coordinates of ( $q$, $v)$ in $T U$, it is easy to obtain a differential structure for $T M$. The tangent bundle $T U=U$ $\times \mathbb{R}^{n}$ is locally a product. The canonical projection $\pi: T M \rightarrow M$ given by $\pi(q, v)=q$ is differentiable.

Theorem 2.3 If $X$ is a $C^{\infty}$ vector field on the open set $V$ in the manifold $M$ and $p \in V$ then there exist an open set $V_{o} \subset V, p \in V$, a number $\delta>0$ and a $C^{\infty}$ mapping $\phi:(-\delta, \delta) \times V_{o}$ $\rightarrow V$ such that the curve $t \rightarrow \phi(t, q), t(-\delta, \delta)$ is the unique trajectory of $X$ which at the instant $t=0$ passes through the point $q$, for every $q \in V_{o}$ ( do Carmo 1976 ).
The mapping $\phi_{t}: V_{0} \rightarrow V$ given by $\phi_{t}(q)=$ $\phi(t, q)$ is called the flow of $X$ on $V$.

Definition 2.4 The vector field $G$ on $T M$ whose trajectories are of the form $t \rightarrow(\gamma(t), \gamma$ $(t)$ ) is called the geodesic field on TM and its flow is called the geodesic flow on TM.

Proposition 2.5 Given $p \in M$, there exist an open set $V \subset M, p \in V$, number $\delta>0$ and $\epsilon_{1}$ $>0$ and a $C^{\infty}$ mapping

$\gamma:(-\delta, \delta) \times \mathrm{U} \rightarrow M, \mathrm{U}=\left\{(q, v) \mid q \in V, v \in T_{q} M\right.$, $\left.|v|<\epsilon_{1}\right\}$,

such that the curve $t \rightarrow \gamma \quad(t, q, v), t \in(-\delta, \delta)$ is the unique geodesic of $M$ which at the instant $t=0$, passes through $q$ with velocity $v$, for each $q \in V$ and for each $v \in T_{q} M$ with $|\mathrm{v}|<\epsilon_{1}$.

Lemma 2.6 (Rauch 1953) (Homogeneity of a geodesic) If the geodesic $\gamma(t, q, v)$ is defined on the interval $(-\delta, \delta)$ then the geodesic $\gamma(t, q, a v) a \in \mathbb{R} a>0$, is defined on the interval $\left(-\frac{\delta}{a}, \frac{\delta}{a}\right)$ and $\gamma(t, q, a v)=\gamma(a t, q, v)$

Definition 2.7 Let $p \in M$ and let $\mathrm{U} \subset T M$ be an open set given by $U=\{(q, v) \in T M \mid$ $\left.q \in V, v \in T_{q} M,|v|<\in\right\}$. Then the map exp : $\mathrm{U} \rightarrow M$ given by

$\exp (q, v)=\gamma(1, q, v)=\gamma\left(|v|, v, \frac{v}{|v|}\right),(q, v) \in \mathrm{U}$ is called the exponential map on $\mathrm{U}$.

Clearly, exp is differentiable. In the application we shall use the restriction of exp to 
an open subset of the tangent space $T_{q} M$ and we define

$$
\exp _{q}: \mathrm{B}_{\in}(0) \subset T_{q} M \rightarrow \mathrm{M}
$$

by $\exp _{q}(v)=\exp (q, v)$, where $B_{\in}(0)$ is an open ball with center at the origin $\mathrm{O}$ of $T_{q} M$ and of radius $\in$. We can prove that $\exp _{q}$ is differentiable and $\exp _{q}(0) q$.

Proposition 2.8 Given $q \in M$, there exists an $\in>0$, such that $\exp _{q}: B_{\in}(0) \subset T_{q} M \rightarrow \mathrm{M}$ is a diffeomorphism of $B_{\epsilon}(0)$ onto an open subset of $M$.

\section{Minimizing Properties of Geodesics}

Definition 3.1 A piecewise differentiable curve is a continuous mapping $c:[a, b] \rightarrow$ $M$ of a closed interval $[a, b] \subset \mathbb{R}$ into M satisfying the following condition : there exists a partition $a=t_{0}<t_{1}<\ldots t_{k-1}<t_{k}=b$ of $[a, b]$ such that the restrictions $c \mid\left[t_{1 .} t_{1+1}\right], i=$ $0, \ldots, k-1$ are differentiable. We say that $c$ joins the points $c(a)$ and $c(b) . \quad c\left(t_{1}\right)$ is called a vertex of $c$ and the angle formed by

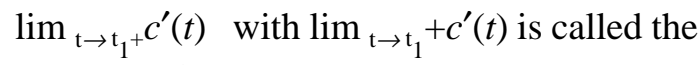
vertex angle at $c\left(t_{1}\right)$.

Lemma 3.2 ( do Carmo 1976 ) (Symmetry) If $M$ is a differentiable manifold with a symmetric connection and $\mathrm{S}: \mathrm{A} \subset\left[\mathrm{R}^{2} \rightarrow \mathrm{M}\right.$ is a parametrized surface then

$$
\frac{D}{\partial v} \frac{\partial S}{\partial U}=\frac{D}{\partial u} \frac{\partial S}{\partial v}
$$

Lemma 3.3 ( Rauch 1953 ) (Gauss) Let $p \in$ $M$ and let $v \in T_{p} M$ such that $\exp _{p} v$ is defined.
Let $\quad w \in T_{p} M \sim T_{v}\left(T_{p} M\right)$. Then

$<\left(\operatorname{dexp}_{p}\right)_{v}(v),\left(\operatorname{dexp}_{p}\right)_{v}(w)>=<v, w$

Proof. Let $w=w_{T}+w_{N}$ where $w_{T}$ is parallel to $v$ and $w_{N}$ is normal to $v$. Since $\operatorname{dexp}_{p}$ is linear and by the definition of $\exp _{p}$, it suffices to prove (1) for $w=w_{N}$ It is clear that we can assume $w_{N} \neq 0$.

Since $\exp _{p} v$ is defined, there exists $\in>0$ such taht $\exp _{p} u$ is defined for

$$
u=t v(s), \quad o \leq t \leq 1, \quad-\in<s<\epsilon,
$$

where $v(s)$ is a curve in $T_{p} M$ with $v(0)=v$, $v^{\prime}(0)=w_{N}$ and $|v(s)|$ constant. Now, we can consider the parametrized surface (Ahmed 2004 )

$f: A \rightarrow M, A=\{t, s) \mid 0 \leq t \leq 1,-\in<s<\epsilon\}$

given by $f(t, s)=\exp _{p} t v(s)$.

Observe that the curve $\mathrm{t} \rightarrow f\left(t, \mathrm{~s}_{0}\right)$ are geodesics.

$\frac{\partial f}{\partial t}=\left(d \exp _{p}\right)_{t v(s)} v(s), \quad \frac{\partial f}{\partial s}=\left(d \exp _{p}\right)_{t v(s)} t v^{\prime}(s)$

To prove (1) for $w=w_{N}$ observe first that by putting $t=1, s=0$

$<\frac{\partial f}{\partial s}, \frac{\partial f}{\partial t}>(1,0)=\left(d \exp _{p}\right)_{v}\left(w_{N}\right),\left(d \exp _{p}\right),(v)>$

In addition, for all (t, s), we have

$$
\frac{\partial}{\partial t}<\frac{\partial f}{\partial s}, \frac{\partial f}{\partial t}>=<\frac{D}{\partial t} \frac{\partial f}{\partial s}, \frac{\partial f}{\partial t}>+<\frac{\partial f}{\partial s}, \frac{D}{\partial t} \frac{\partial f}{\partial t}>
$$

The last term of the above expression is zero, 
since $\frac{\partial f}{\partial t}$ is the tangent vector of a geodesic. From the symmetry of the connection, the first term of the sum is transformed into

$$
<\frac{D}{\partial t} \frac{\partial f}{\partial s}, \frac{\partial f}{\partial t}>=<\frac{D}{\partial s} \frac{\partial f}{\partial t}, \frac{\partial f}{\partial t}>=\frac{1}{2} \frac{\partial}{\partial s}<\frac{\partial f}{\partial t}, \frac{\partial f}{\partial t}>=0
$$

It follows that $<\frac{\partial f}{\partial s}, \frac{\partial f}{\partial t}>$ is independent of $t$. Since

$$
\lim _{t \rightarrow 0} \frac{\partial f}{\partial s}(t, o) \lim _{t \rightarrow 0}\left(d \exp _{p}\right)_{t v} t w_{N}=0
$$

we conclude that $<\frac{\partial f}{\partial s}, \frac{\partial f}{\partial t}>(1,0)=0 \quad$ which together with (2) proves the lemma.

\section{The Derivation of Jacobi Field Equation}

Let $M$.. be a Riemannian manifold and let $p \in M \quad$ In Gauss Lemma we saw that if $\exp _{p}$ is defined at $v \in T_{p} M$ and if $w \in T_{v}\left(T_{p} M\right)$ then

$$
\left(d \exp _{p}\right)_{v} w=\frac{\partial f}{\partial s}(1,0),
$$

where $\mathrm{f}$ is a parametrized surface given by $f(t, s)=\exp _{p} t v(s), \quad 0 \leq t \leq 1, \quad-\in \leq s \leq \epsilon$ and $v(\mathrm{~s})$ is a curve in $T_{p} M$ with $v(0)=v$, $v^{\prime}(0)=w$.

It is convenient to extend our object slightly and study the field

$\left(d \exp _{p}\right) t v(t w)=\frac{\partial f}{\partial s}(t, 0)$

along the geodesic $\gamma(t)=\exp _{p}(t v), \quad 0 \leq t \leq 1$. The remark is that $\frac{\partial f}{\partial s}$ satisfies a differential equation. Since $\gamma$ is a geodesic, we have $\frac{D}{\partial t} \frac{\partial f}{\partial t}=0$, for all $(t, s)$.
Lemma 4.1 Let $f: \mathrm{A} \subset\left[\mathrm{R}^{2} \rightarrow M\right.$ be parametrized surface and let $(t, s)$ be the usual coordinates of [ $\mathrm{R}^{2}$ Let $V=V(t, s)$ be a vector field along $f$. For each $(t, s)$, it is possible to define $R\left(\frac{\partial f}{\partial s}, \frac{\partial f}{\partial t}\right)$ in an obvious following manner:

$$
\frac{D}{\partial t} \frac{D}{\partial s} V-\frac{D}{\partial s} \frac{\partial}{\partial t} V=R\left(\frac{\partial f}{\partial s}, \frac{\partial f}{\partial t}\right) V
$$

Proof. Consider a system ( U, x ) based at $p \in M$ Let

$$
V=\sum_{i} v^{i} X_{i}
$$

where $v^{i}=v^{i}(s, t)$ and $X_{i}=\frac{\partial}{\partial x_{i}}$. Then

$\frac{D}{\partial s} V=\frac{D}{\partial s}\left(\sum_{i} v^{i} X_{i}\right)=\sum_{i} v^{i} \frac{D X_{i}}{\partial s}+\sum \frac{D v^{i}}{\partial s} X_{i}$ and

$\frac{D}{\partial t}\left(\frac{D}{\partial s} V\right)=\sum_{i} v^{i} \frac{D}{\partial t} \frac{D X_{i}}{\partial s}+\sum_{i} \frac{\partial v^{i}}{\partial t} \frac{D X_{i}}{\partial s}+$

$$
\sum_{i} \frac{\partial v^{i}}{\partial s} \frac{D x_{i}}{\partial t} X_{i}+\sum_{i} \frac{\partial^{2} v^{i}}{\partial t \partial s} X_{i}
$$

By interchanging the coordinates $\mathrm{s}$ and $\mathrm{t}$ in the above expression, we obtain

$$
\begin{aligned}
& \frac{D}{\partial s}\left(\frac{D}{\partial t} V\right)=\sum_{i} v^{i} \frac{D}{\partial s} \frac{D X_{i}}{\partial t}+\sum_{i} \frac{\partial v^{i}}{\partial s} \frac{D X_{i}}{\partial t} \\
& \sum_{i} \frac{\partial v^{i}}{\partial t} \frac{D x_{i}}{\partial s} X_{i}+\sum_{i} \frac{\partial^{2} v^{i}}{\partial s \partial t} X_{i}
\end{aligned}
$$


Now, subtracting (5) from (4), we obtain

$$
\begin{aligned}
& \frac{D}{\partial t}\left(\frac{D}{\partial s} V\right)-\frac{D}{\partial s}\left(\frac{D}{\partial t} V\right) \\
& =\sum_{i} v^{i}\left(\frac{D}{\partial t} \frac{D X_{i}}{\partial s}-\frac{D}{\partial s} \frac{D X_{i}}{\partial t}\right)
\end{aligned}
$$

since, $\sum_{i} \frac{\partial v^{i}}{\partial s} \frac{D X_{i}}{\partial t}=\sum_{i} \frac{\partial v^{i}}{\partial t} \frac{D X_{i}}{\partial s} \quad \&$

$$
\sum_{i} \frac{\partial^{2} v^{i}}{\partial t \partial s} X_{i}=\sum_{i} \frac{\partial^{2} v^{i}}{\partial s \partial t} X_{i}
$$

Next, we need to calculate $\frac{D}{\partial t} \frac{D X_{i}}{\partial s}$.

Put $f(s, t)=\left(x_{1}(s, t), x_{2}(s, t), \ldots, x_{n}(s, t)\right)$.

Then $\frac{\partial f}{\partial s}=\sum_{j} \frac{\partial x_{j}}{\partial s} X_{j}$ and

$$
\frac{\partial f}{\partial t}=\sum_{k} \frac{\partial x_{k}}{\partial t} X_{j}
$$

where $X_{j}=\frac{\partial}{\partial x_{j}}$ and $X_{k}=\frac{\partial}{\partial x_{k}}$. Thus we calculate

$$
\begin{aligned}
& \frac{D X_{i}}{\partial s}=\nabla_{\sum_{j} \frac{\partial x_{j}}{\partial s} X_{j}} X_{i}=\sum_{j} \frac{\partial x_{j}}{\partial s} \nabla_{X_{j}} X_{i} \\
& \text { and } \frac{D}{\partial t} \frac{D X_{i}}{\partial s}=\frac{D}{\partial t}\left(\sum_{j} \frac{\partial x_{j}}{\partial s} \nabla_{X_{j}} X_{i}\right) \\
& =\sum_{j} \frac{\partial^{2} x_{j}}{\partial t \partial s} \nabla_{X_{j}} X_{i}+\sum_{j} \frac{\partial x_{j}}{\partial s} \nabla_{\sum_{k}} \frac{\partial x_{k}}{\partial t} X_{k}\left(\nabla_{X_{j}} X_{i}\right) \\
& =\sum_{j} \frac{\partial^{2} X_{j}}{\partial t \partial s} \nabla_{X_{j}} X_{i}+\sum_{j} k \frac{\partial x_{j}}{\partial s} \frac{\partial x_{k}}{\partial t} \nabla_{X_{k}}\left(\nabla_{X_{j}} X_{i}\right)
\end{aligned}
$$

In the similar way, we obtain

$$
\begin{aligned}
& \frac{D}{\partial s} \frac{D X_{i}}{\partial t}=\sum_{j} \frac{\partial^{2} x_{j}}{\partial s \partial t} \nabla_{X_{j}} X_{i}+ \\
& \sum_{k} j \frac{\partial x_{k}}{\partial t} \frac{\partial x_{j}}{\partial s} \nabla_{X_{j}}\left(\nabla_{X_{k}} X_{i}\right)
\end{aligned}
$$

Subtracting (9) from (8), we obtain

$$
\begin{aligned}
& \left(\frac{D}{\partial t} \frac{D}{\partial s}-\frac{D}{\partial s} \frac{D}{\partial t}\right) X_{i}= \\
& \sum_{j} k \frac{\partial x_{j}}{\partial s} \frac{\partial x_{k}}{\partial t}\left(\nabla_{X_{k}}\left(\nabla_{X_{j}} X_{i}\right)-\nabla_{X_{j}}\left(\nabla_{X_{k}} X_{i}\right)\right) \\
& =\sum_{j k} \frac{\partial x_{j}}{\partial s} \frac{\partial x_{k}}{\partial t} R\left(X_{j}, X_{k}\right) X_{i}
\end{aligned}
$$

[using def. of curvature [1]]

$\sum_{i} v^{i}\left(\frac{D}{\partial t} \frac{D}{\partial s}-\frac{D}{\partial s} \frac{D}{\partial t}\right) X_{i}=$

$\sum_{i j k} v^{i} \frac{\partial x_{j}}{\partial s} \frac{\partial x_{k}}{\partial t} R\left(X_{j}, X_{k}\right) X_{i}$

$=R\left(\sum_{j} \frac{\partial x_{j}}{\partial s} X_{j}, \sum_{k} \frac{\partial x_{k}}{\partial t} X_{k}\right) \sum_{i} v^{i} X_{i}$

$=R\left(\frac{\partial f}{\partial s}, \frac{\partial f}{\partial t}\right) V \quad$ [using (3) and (7)]

Hence, (6) implies that

$\frac{D}{\partial t} \frac{D}{\partial s} V-\frac{D}{\partial s} \frac{\partial}{\partial t} V=R\left(\frac{\partial f}{\partial s}, \frac{\partial f}{\partial t}\right) V$

Now, the lemma 4.1 gives

$\frac{D}{\partial t}\left(\frac{D}{\partial s} \frac{\partial f}{\partial t}\right)-R\left(\frac{\partial f}{\partial s}, \frac{\partial f}{\partial t}\right) \frac{\partial f}{\partial t}=\frac{D}{\partial s}\left(\frac{D}{\partial t} \frac{\partial f}{\partial t}\right)$

$\frac{D}{\partial t} \frac{D}{\partial s} \frac{\partial f}{\partial t}+R\left(\frac{\partial f}{\partial t}, \frac{\partial f}{\partial s}\right) \frac{\partial f}{\partial t}=0$ 
Putting $\frac{\partial f}{\partial s}(t, 0)=J(t)$, we obtain that $J$ satisfies the equation

$$
\frac{D^{2} J(t)}{\partial t^{2}}+R\left(\gamma^{\prime}(t), J(t)\right) \gamma^{\prime}(t)=0
$$

and the equation (10) is known as the Jacobi equation with the relation between curvature and geodesics of a Riemanian manifold $M$.

Definition 4.2 Let $\gamma:[0, a] \rightarrow M$ be a geodesic in $M$ ( Kulkarni 1970 ). A vector field $J$ along $\gamma$ is said to be a Jacobi field if it satisfies the Jacobi equation (10), for all $t \in$ $[0, a]$.

A Jacobi field is determined by its initial conditions $J(0), \frac{D J(o)}{d t}$. Let $e_{1}(\mathrm{t}) e_{2}(\mathrm{t}), . ., e_{\mathrm{n}}(t)$ be parallel, orthonormal fields along $\gamma$, we shall write (Ahmed 2004 )

$J(t)=\sum_{i} f_{i}(t) e_{i}(t)$

$$
a_{i j}=<R\left(\gamma^{\prime}(t), e_{i}(t)\right) \gamma^{\prime}, e_{j}(t)>,
$$

$i, j=1,2, \ldots, n=\operatorname{dim} M$.

$$
\text { Then } \frac{D^{2} J}{d t^{2}}=\sum_{i} f_{i}^{\prime \prime}(t) e_{i}(t)
$$

and $R\left(\gamma^{\prime}, J\right) \gamma^{\prime}=\sum_{j}<R\left(\gamma^{\prime}, J\right) \gamma^{\prime}, e_{j}>e_{j}$ $=\sum_{i j} f_{i}<R\left(\gamma^{\prime}, e_{i}\right) \gamma^{\prime}, e_{j}>e_{j}$ [since $J=\sum_{i} f_{i} e_{i}$ ]

$$
=\sum_{i j} f_{i} a_{i j} e_{j}
$$

Therefore, the equation (10) is equivalent to the system

$$
f_{j}^{\prime \prime}(t)+\sum_{i} a_{i j} f_{i}(t)=0, \quad j=1,2, \ldots, n
$$

which is a linear system of the second order. For the given initial conditions $J(0), \frac{D J(0)}{d t}$, there exists a $C^{\infty}$ solution of the system defined on [0,a] and 2nd linearly independent Jacobi fields along $\gamma$.

Remark 4.3 We observe that $\gamma(e)$ and $t$ $\gamma($ (e) are Jacobi fields along $\gamma$. The first field has derivative zero and vanishes and the second field is zero if and only if $t=0$. For these reason, we shall consider Jacobi fields along $\gamma$ that are normal to $\gamma(\mathrm{C}$

Lemma 4.4 Let $M$ be a Riemannian manifold and $p$ be a point of $M$. Define a tri-linear mapping (Ahmed 2003) R' : $T_{p} M \times T_{p} M^{\prime}$ $\times T_{p} M \rightarrow \times T_{p} M$ by

$<R^{\prime}(X, Y, W), Z>=<X, W><Y, Z>-$ $<Y, W><X, Z>$,

for all $X, Y, W, Z_{\in} T_{p} M$. Then $M$ has constant sectional curvature equal to $K$ if and only if $R=K R$, where $R$ is the curvature of $M$.

Using the lemma 4.4, we have for all vectors 
$T$ along $\gamma$

$$
\begin{aligned}
& <R\left(\gamma^{\prime}, J\right) \gamma^{\prime}, T>=K<R^{\prime}\left(\gamma^{\prime} J\right) \gamma^{\prime}, T> \\
& =K\left\{<\gamma^{\prime}, \gamma^{\prime}><J, T>-<J, \gamma^{\prime}><\gamma^{\prime}, T>\right\} \\
& =K<J, T>
\end{aligned}
$$

[since $\left\langle\gamma^{\prime}, \gamma^{\prime}\right\rangle=1$ and $\left\langle\gamma^{\prime}, T\right\rangle=0$ ]

Hence, $R(\gamma, J) \gamma^{\prime}=K J$

As a result, the Jacobi equation can be written as

$$
\frac{D^{2} J}{d t^{2}}+K J=0
$$

which is the standard form of Jacobi equation with a Jacobi field $J$ and a constant sectional curvature $K$.

Let $w(\mathrm{t})$ be a parallel field along $\gamma$ with $<\gamma^{\prime}(t)$, $w(t)>=0$ and $|w(t)|=1$.It is easy to verify that

$$
J(t)=\left\{\begin{array}{llc}
\frac{\sin (t \sqrt{K})}{\sqrt{K}} & \text { if } & K>0, \\
t w(t) & \text { if } & K=0, \\
\frac{\sinh (t \sqrt{-K})}{\sqrt{-K}} & \text { if } & K<0,
\end{array}\right.
$$

is a solution of (12) with initial conditions $J(0)=0, J^{\prime}(0)=w(0)$.

\section{Conclusion}

A new approach of Jacobi field equation and its solution have been derived in Riemannian manifolds by using exponential mapping, geodesics, Gauss lemma. Using this Jacobi field equation and its solution along geodesic $\gamma$, one can find expansion of $|J(t)|^{2}=\langle J(t)$, $J(t)\rangle$ about $t=0$, the value of $\langle J(t), \gamma(t)\rangle$ and other results which are involved with Riemannian curvature $R$.

\section{References}

Ahmed, K.M. (2003). Curvature of a Riemannian manifold, Ganit: J. Bangladesh Math. Soc., 22: 25-29.

Ahmed, K.M. (2004). Jacobi fields along geodesics in Riemannian Geometry, Dhaka Univ. J. Sci. 52(2): 233- 240.

Do Carmo, M. (1976). Differentiable Curves and Surfaces, Prentice-Hall, New Jersey.

Gauss, K.F. (1965). General Investigations of Curved Surfaces, Raven Press, New York.

Kulkarni, R. (1970). Curvature and metric, Ann. of Math. 99: 311-331.

Klingenberg, W. (1959). Conrtributions to Riemannian Geometry in the large, Ann. of Math. 69: 654- 666. 
Myers, S.B. (1941). Riemann manifolds with positive mean curvature, Duke Math. J. 8: 401-404.

Riemann, B. (1959). On the hypotheses which lie at the foundations of geometry, translated from the German by Henry S. White In: A Source Book in Mathematics, by David E Smith, Dover edition, 2: Dover, New York.
Rauch, H.E. (1953). Geodesics, symmetric spaces and differential geometry in the large, Comment. Math. Helv. 27: 294-320.

Received : May, 15, 2008;

Accepted : August 04, 2008 